\title{
Interaction of electron beam and gold nanoparticles
}

Cuauhtémoc Núñez Valencia ${ }^{1}$, Matthew Helmi Leth Larsen ${ }^{1}$, William Bang Lomholdt ${ }^{1}$, Pei Liu ${ }^{1}$, Thomas Hansen $^{2}$ and Jakob Schiøtz ${ }^{3}$

${ }^{1}$ Technical University of Denmark, United States, ${ }^{2}$ DTU Nanolab, Technical University of Denmark, Kgs. Lyngby, Hovedstaden, Denmark, ${ }^{3}$ Department of Physics, Technical University of Denmark, Kongens Lyngby, Hovedstaden, Denmark

Beam damage in High-Resolution Transmission Electron Microscopy (HRTEM) is poorly understood theoretically, and not yet well described phenomenologically. For example, it is not yet known whether it is the total dose (electrons per area) or the dose rate (electrons per area per time) that is most critical for inducing beam damage [1,2]. In order to study the dependence of the total dose, molecular dynamics simulation can be an appropriate tool to understand the atomic level behavior, when coupled to experimental observations.

Using molecular dynamics, we study the beam damage in gold nanoparticles caused by high-energy electron beam irradiation. We assume that the main damage mechanism in metallic nanoparticles is knock-on damage, where an energetic electron transfer momentum and energy to an atom through Rutherford scattering.

In this work, we focus on the simplest mechanism for beam damage: direct transfer of momentum between the energetic electrons and the nuclei through Rutherford scattering. Several molecular dynamics studies have previously focus on electron head-on collision in carbon nanomaterials $[3,4]$. Here, we extend these methods to include collisions with non-zero impact parameter, and build a model including both complete removal of atoms as well as beam-induced surface diffusion on the nanoparticle (figure 1 shows an atom removed from its initial position on a gold nanoparticle), in the context of HRTEM. We use a stochastic approach for the momentum transfer to the atoms in the nanoparticle, focusing on momentum transfer to the easily removed surface atoms. In this way, for each nanoparticle size, we get a rate of atom removal and a rate of induced surface diffusion proportional to the beam intensity. We compare these rates with observation of beam induced surface diffusion and beam induced damage in gold nanoparticles on a cerium dioxide $(\mathrm{CeO} 2)$ substrate, as observed in HRTEM (see figure 2). The analysis of the experimental data leverages convolutional neural networks to identify when the structure changes [5].

In opposition to the total dose, effects of the dose rate are expected to be shown predominantly through the heating of the nanoparticle due to the increase of the events per unit of time as we increase the dose rate. In addition to heating from the many Rutherford scattering events with too low momentum transfer to inflict direct damage, there may also be a heating effect due to interactions between the beam electrons and the conduction electrons of the metals. Simple models can be made for these effects, and rough estimates of the heating of the nanoparticle can then be used to extend the current molecular dynamics simulations to higher temperatures, where beam damage will occur more readily. 

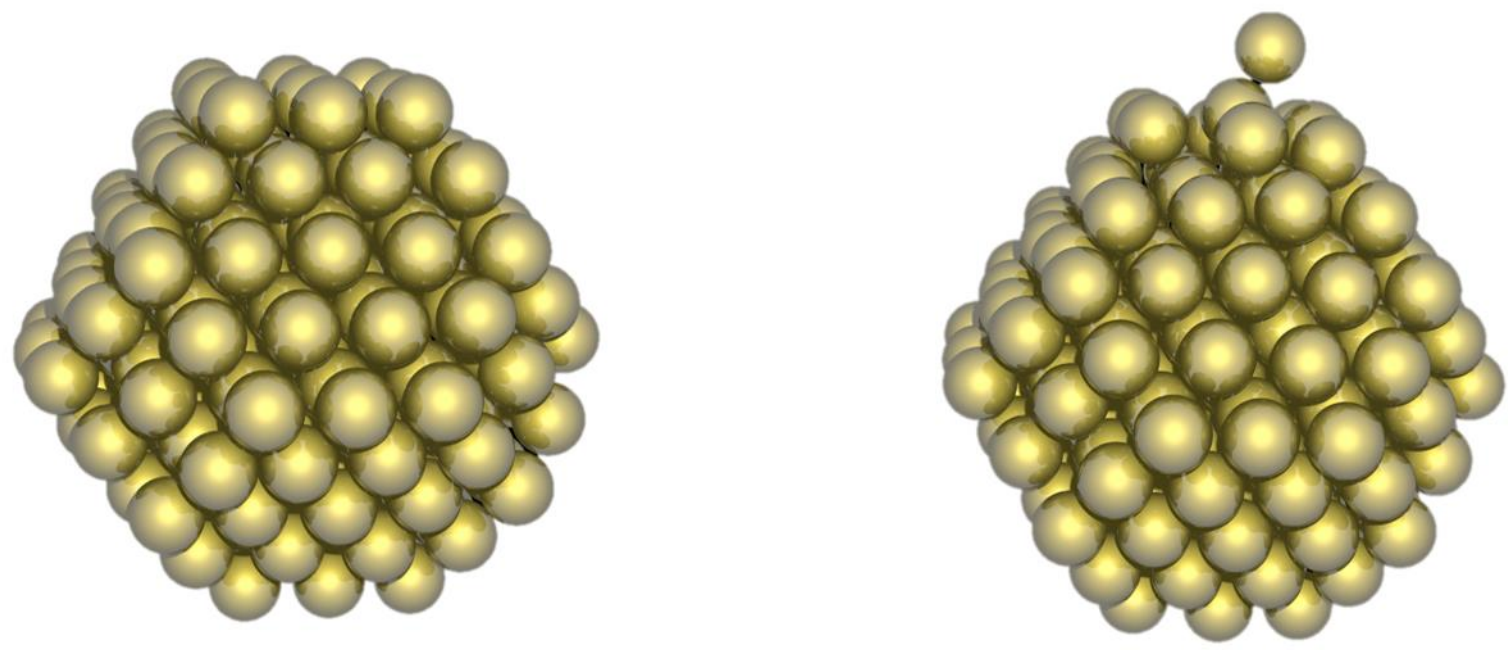

Figure 1. Figure 1: Molecular dynamics simulation gold nanoparticle before (left) and after an atom has been kicked by the high-energy electron beam (right). Initially the gold atom at the surface of the nanoparticle leaves its position due to the momentum gained by electron irradiation at $300 \mathrm{keV}$. The temperature of the nanoparticle before the electron irradiation was kept constant at $300 \mathrm{~K}$.
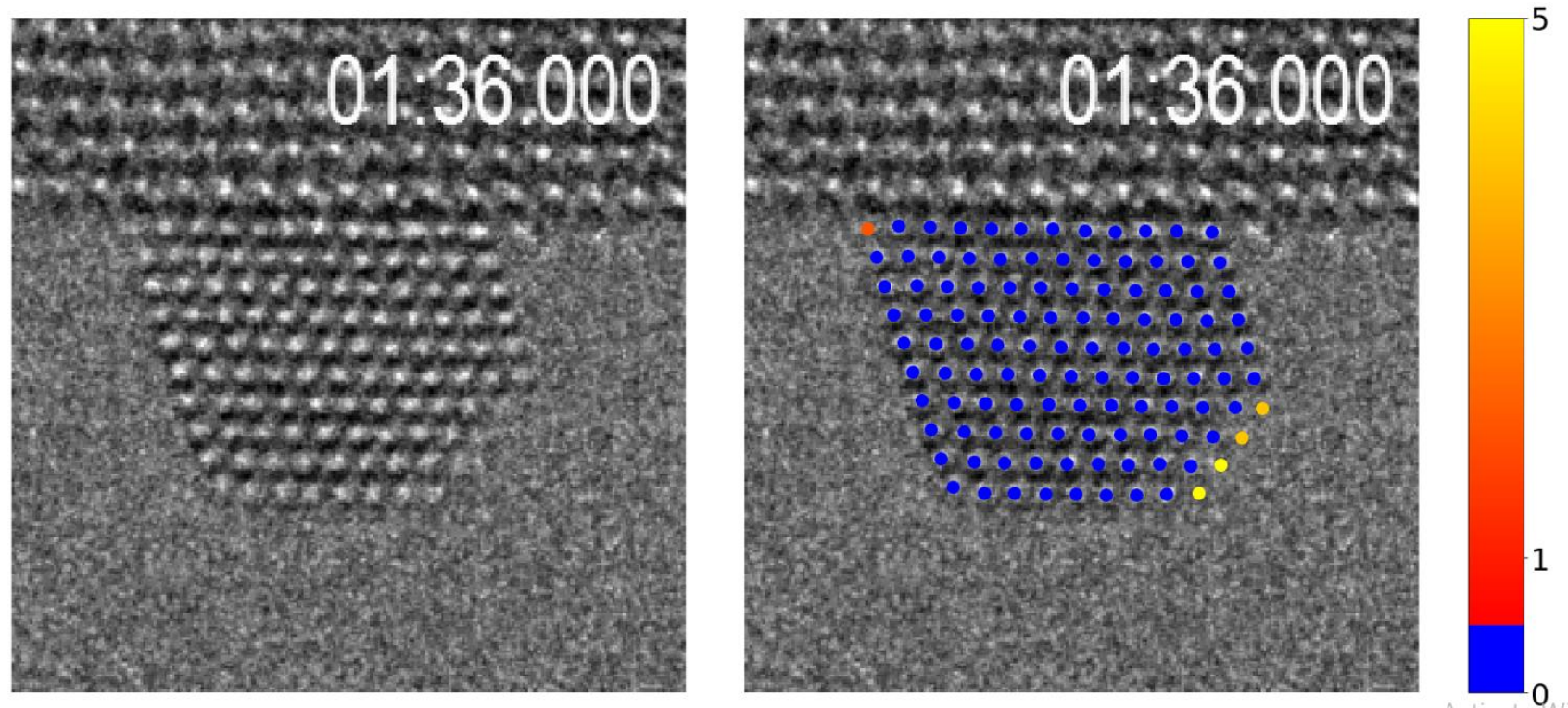

Figure 2. Figure 2: Analysis of an image sequence for surface diffusion (raw image left and analyzed image right). A neural network has identified the positions of all atom columns in the nanoparticle during the sequence. This sequence has then been analyzed further to count the number of events where a whole column of atoms appears or disappears. The resulting analysis is indicated by a colored circle at the position of the atomic column. One surface is particularly active, where the atomic columns are appearing and disappearing up to five times during the 1.5 minutes duration of the image sequence. The images show gold nanoparticle on $\mathrm{CeO} 2$ substrate, in an atmosphere of $4.5 \mathrm{~Pa}$ carbon monoxide (CO) at a temperature 
of $200^{\circ} \mathrm{C}$. Images are recorded using an aberration corrected $300 \mathrm{keV}$ FEI Titan ETEM. The color bar represents the dynamic events in the analyzed image.

\section{References}

[1] Kisielowski, Christian, et al. "Real-time sub-Ångstrom imaging of reversible and irreversible conformations in rhodium catalysts and graphene." Physical Review B 88.2 (2013): 024305.

[2] Kisielowski, C., et al. "Detecting structural variances of Co 3 O 4 catalysts by controlling beam-induced sample alterations in the vacuum of a transmission electron microscope." Advanced structural and chemical imaging 2.1 (2016): 1-14.

[3] Yasuda, Masaaki, et al. "Interaction volume of electron beam in carbon nanomaterials: A molecular dynamics study." MRS Online Proceedings Library 1700.1 (2014): 29-35.

[4] Pregler, Sharon K., and Susan B. Sinnott. "Molecular dynamics simulations of electron and ion beam irradiation of multiwalled carbon nanotubes: The effects on failure by inner tube sliding." Physical Review B 73.22 (2006): 224106.

[5]. Madsen, Jacob, et al. "A deep learning approach to identify local structures in atomic-resolution transmission electron microscopy images." Advanced Theory and Simulations 1.8 (2018): 1800037. 